introduction of a fillet or tube-canula through the opening into the nose. This is substantially the treatment followed to-day.

With the signs and symptoms of a tumor in the locality mentioned we would be inclined to make an exploratory incision. A small exploring needle or a very small drill would give important information as to the contents and thickness of the bony wall of these swellings, if the case presented itself before absorption of the bone has taken place. If a collection of pus be found, a trephine, or the dental engine's drills or a chisel should be freely used. After an opening large enough to admit the finger has been made over the site of the disease and careful exploration of the cavity has taken place, a free opening should be made into the nose, the instrument, guided by a finger of the left hand, introduced into the nostril of the same side. If necessary, force may be used in breaking through the floor of the cavity into the upper part of the nose. A perforated rubber drainage tube should be introduced into the nose through the opening and the entire cavity washed with some antiseptic fluid. Listerine proved very valuable and pleasant in our case. An astringent may be used later, if necessary.

In the records of the cases analyzed one is at once struck by the comparatively small amount of attention paid to the subject of free drainage through the nose. In six cases no mention is made of this, and of these only one is said to have been cured. It is stated in eleven cases that there was no drainage through the nose; of this number three were cured, seven not cured, one not mentioned. In twenty-five cases in which there was free drainage in to the nose twenty-three were cured, and in the other two the result was not stated.

Soelberg Wells advises passing an instrument upwards from the nose into the sinus. Ogston ${ }^{22}$ says he has never been able to do so, and in our case it was tried, but was found absolutely impracticable. Except in favorable cases this would seem to be very difficult.

In cleaning the cavity, naturally any foreign body would be removed; and in case of polypoid degeneration of the mucous membrane lining the sinus, as described by Ogston and $\mathrm{Pé}^{28}{ }^{28}$, this should be thoroughly destroyed. Any dead bone should be removed, using great care and no force if the bone be connected with the posterior wall of the sinus, lest the membranes of the brain or the brain itself should be injured.

Death occurred in six cases ; in four caused by secondary abscess of the brain, in one by meningitis, and in another by albuminuria.

For help in the preparation of this article we are much indebted to Dr. C. J. Colles. The greater number of references has been omitted purposely,

\footnotetext{
22 Ogston. Med. Chronicle, Manchester, $1884-5$, i, p. 235

23 Péan. Gaz. des Hop., Paris, 1881, p. 66.
}

as some of the later German articles and French theses are not obtainable. An addendum will be published containing references to these and bibliography to date.

DR. JONATHAN WRIGHT reported a case in an old woman in whom, after removal of intranasal obstruction, it was necessary to trephine the supraorbital sinus, and this still proving unavailing, the whole anterior wall was removed and the wound allowed to heal by granulation from the bottom. When last seen this procedure promised success, but a large and very deforming cicatrix must result, though drainage was of course resorted to, both after trephining and after the more extended operation.

\section{INITIAL EYE SYMPTOMS IN LOCOMOTOR ATAXY.}

Kead before the Fort Wayne Academy of Medicine.

BY KENT K. WHEELOCK, M.D., OF FORT WAYNE, IND.

PROFESSOR OF OPHTHALMOLOGY AND OTOLOGY IN THE FORT WAYNE COLLEGE OF MEDICINE.

The case which I am about to present for your consideration involves the question of the prodromal symptoms of locomotor ataxy, and while to my mind the symptoms are sufficient to warrant me in arriving at the conclusion that my patient has the prodromata of tabes, yet if any one should think the case not fully established his fullest criticism is invited as much in the interest of the patient as the discussion of the whole question involved.

On November 27,1887 , Frederick B., æt 55, iron merchant in Fort Wayne, consulted me for an ear trouble which had bothered him for some time, as much on account of a singing noise which had recently sprung up as the defect in hearing which had annoyed him for some time. The singing was confined to the left ear.

Right ear W. $=\frac{3}{36}$, left ear W. $=$ P. Eustachian tube not open to catheter, Politzer or Valsalvas' method. By traction on membrana tymp. with Siegel's otoscope there was some improvement in the noise. Treatment by attempted inflation, catheterization and local application did not improve the hearing or noise so the patient was not recommended to continue.

On June 21, I889, he again came under my observation and gave me the following history: Has suffered from occasional frontal headache for three years, vomiting on two or three occasions Headaches came on about every three or four weeks and lasted from twelve to eighteen hours. On May ist of the present year had a serious headache which culminated in vomiting at about 3 P.M.; at the same time patient lost consciousness and fell to the floor. He remained unconscious. five minutes, the eyes turning up, but not oscil- 
lating. Has had dizzy spells for about one year, and the dizziness has increased in intensity till at present are frequent, depending upon his occupation. When he looks up or ascends a stair it becomes worse. The increase has been greater since May I. Has had sharp shooting pains in the legs below the knees for a year or two, which were attributed to rheumatism, and were treated as such. Yet he had no joint swelling or painful joints. The trouble for which I was consulted was double vision, which came on after the attack in May. He consulted a quack first and was under his treatment for six weeks.

Upon examination I found right eye S. $\frac{20}{30} ;+$ $0.5 \mathrm{D} . \mathrm{S} .=\frac{20}{2}$; left eye $=\frac{20}{20} ;$ accepts $0.5 \mathrm{D}$. Right eye +2.5 D. Jaeger No. I, I 2-I 6 ; left eye same, showing that the accommodation ruled by the third nerve is not impaired. The double images are vertical and when neither eye is covered with a colored glass the image of the left eye stands $2 \frac{1}{2}$ feet above the image of the right eye, and leans towards the perpendicular or lower one, showing that the cornea of the left eye looks downwards when the candle flame impinges on the retina below the macula and is projected in the same direction it would project images if the optic axis were horizontal or parallel with the other eye. The movement of both eyes is perfect in every direction, showing the effect to be partial only. By placing a prism of $15^{\circ}$ base toward the paretic or superior rectus muscle, the images are single, or $8^{\circ}$ base up before left eye and $7^{\circ}$ base down before right eye blend the images. The Argyle-Robertson puyil is present; the pupil is about the size of a pin head and does not respond to stimulation of light, either artificial or natural, when intensified by condensation. Upon covering either eye and asking the patient to read, the pupils contract ; they also contract when both eyes attempt to converge in the act of read. ing. Under atropia the left pupil dilated in an ovoid manner, but after a few hours the space became circular.

Examination of the ears shows that the t. f. $c^{\prime}$ is not heard in the left ear when placed on the vertex, and the same tone heard distinctly in right ear is not heard at all in left ear; $t$. f. on left mastoid not heard, while the same sound is heard eighteen seconds on the right mastoid in a corresponding position. When struck upon a hard substance, producing a very loud tone, the vibrations heard for a moment at a point near the center of the mastoid and on the temporal bone directly above the external auditory meatus, Voice sound heard very feebly in left ear, W. not at all. Right ear moderate voice heard, also watch. The knee-jerk is exaggerated, and if the patient attempts to close his eyes and stand with his heels together he sways from side to side. His walk backwards is very unsteady and while advancing with eyes open he steps high and drops his feet heavily. While it is a well established clinical fact, substantiated by post-mortem appearances, that deafness of one or both ears frequently follows sclerosis of the posterior columns of the spinal cord, yet tabes could not be predicated upon the presence of this symptom alone, and would only, with our present knowledge, go for a factor in the total symptoms recognized as definitive. Erb regards the coëxistence of the tabes and deafness as accidental rather than causative, and cites Lucas's two cases in support of this statement. Yet he admits the probability of relationship to exist, and cites a case of a Russian naval officer, in which, during the initial stage occasional headaches and deafness had arisen, the latter gradually increasing. This case passed into the hands of Prof. Moos, when, in a few months, total loss of bone conduction was followed by loss of ærial conduction, and the patient became deaf. Later he seems to have modified his opinion, for he states that in some cases of tabes he found definite atrophy of the auditory nerve to be the cause of deafness. Thos. Buzzard, in the Lancet, Sept., I88I, quotes Duchene, Remak and Tobinard as having already pointed out that the auditory nerve may be affected in tabes, and Pierrot showed that it is a frequent complication of this disease. On consulting my notes I find that bone conduction in the case of my lamented friend Dr. J. S. Gregg (who died of tabes), whose ears and eyes I examined a number of times, showed subnormal bone conduction. The late Dr. McBride found a greater or less degree of deafness in every case of tabes which came under his observation, although it was often transitory. In the absence of recorded examinations showing in what the defective hearing consisted, we can only assume that he meant that a subnormal bone conduction existed. Ormerod calls attention to it in five out of thirteen cases. In general I think it can only be regarded as a side light in the picture. Yet when seen in connection with the affections of the third, fourth and sixth the attentive should be at once arrested. Amongst all the affections of the special senses, the disturbance of the muscle bal. ance is most frequent in tabes. As prodromal and transitory symptoms they occur as pupil disturbances and nuscle paresis in more than 50 per cent. of all cases of locomotor ataxy, according to Erb. And it is this transitory character of the eye troubles which establish their value as prodromata. In the course of the disease, and before its termination, some form of eye muscle disturbance occurs in one third of the cases. They then have lost their value and form only another character in the bizzarre picture whose lineaments are so well known. Bunching the observations of ArgyleRobertson, Knapp, Lever and Henpel, the pupils are sometimes contracted or dilated on one side; but more frequently there is a bilateral, uni- 
form, generally pretty considerable contraction, which presents the character of spinal myosis; that is, the pupils do not react to light, but do react distinctly to accommodative impulses. Spitzka says among the exact signs of tabes reflex iridoplegia and abolition of the knee-jerk are the first to appear. He further says that it may be assumed with safety that in 99 out of 100 cases the inability of the pupil to respond to light and the absence of the knee-jerk will be found long before ataxy is developed. Cases have been found where no other positive signs were found. Spitzka found double vision in fifty-eight out of eighty-one tabetic patients in whom the oculo-motor signs were recorded. $\mathrm{He}$ says further: "By far the most important of the exact prodromal signs of tabes are two symptoms-one involving a special faculty of coördination and the other the reflex movement of the pupil. One or both of these must be present to justify the diagnosis of incipient tabes." He further says : "Reflex iridoplegia is, when once established, the most permanent and unvarying evidence of the disease, and is of great differential diagnosis, because it is found in very few other conditions. Spitzka recognizes tinnitus and atrophy of the auditory nerves among the symptoms of tabes." There is one symptom, or sign rather, which a typical tabes should present, absent in my case, viz.: the Westphal symptom. Instead of this loss of reflex my case presents a heightened reflex. This may be accounted for on the supposition that the lateral columns have become affected first, when we have later a combined posterior and lateral sclerosis. It has been claimed that the disappearance of the patellar jerk has been preceded by exaggerated reflex and that the exaggerated reflex is a quite common phenomena.

This paper, read before the Academy of Medicine in Sept., I889, was not intended for publication, but to present what I then thought to be an interesting case of incipient tabes. After referring him back to his family physician with an opinion in accordance with what has been said above, the patient was referred to Prof. Pepper who discovered diabetes mellitus. The eye symptoms were probably due to irritation at the floor of the fourth ventricle, while the ear trouble was due to the spinal trouble as set forth above.

\section{ULCERATIVE ENDOCARDITIS.}

Read in the Section of Practice of Medicine, Maleria Medica and Physiology, at the Fortieth Annual Meeting of the American Medical Association, June, I880.

BY J. G. TRUAX, M.D., OF NEW YORK CITY.

This is not a new disease. It has been known and described under several different names for at least ninety years. It is not the intention of the writer to give a complete history of the disease to

date. Such an effort would be as profitable as digging up the ground in a field thoroughly cultivated and resplendent in the beauty of its production. The writer, in the researches he has made, has failed to discover any really new thought or fact which could be called the fruit of labor in this particular field during the past few years. His intention is to give a history of such cases as have come under his care, together with any thought which may seem to him to be of practical value. Any person desirous of becoming more thoroughly informed on this subject is referred by the writer to Dr. Osler's lecture, published in the Lancet of 1885 , and to the writings of others.

Case I.-Frances Blaza; born in Germany; U. S. five months; age 2 I years; unable to speak English; was admitted into the Harlem Hospital May 31, I887. Her occupation was that of a domestic. No family or personal history could be obtained, excepting the meagre one from her aunt, that her mind was unbalanced at each menstrual epoch, and that her menstrual period did not occur oftener than every three months. $\mathrm{Pa}$ tient was a large, healthy, well-developed girl, and the fact of her not menstruating regularly was evidence that something was wrong with her circulation. When admitted to the hospital she was flowing. Every muscle of her body seemed to take on choreic contractions, and she was unable to remain quiet a moment; mind confused, and at times delirious. The house physician supposed she was stuffering from meningitis, and to give her relief had all the hair shaved from her head and the ice cap applied. He also put her upon a mixture of bromide of potassium and hydrate of chloral. Afterwards she was given sulphate of morphine hypodermically, and quinine in ten grain doses three times daily. These medicines had but little effect. The convulsions lasted to the end.

June ist her highest temperature was $102^{\circ} \frac{3}{5}^{\circ}$; June $2 d, 103 \frac{3}{5}^{\circ}$; pulse, I20; June $3 \mathrm{~d}$, IO $4^{\circ}$; June $4^{\text {th }}, 103 \frac{3}{5}^{\circ}$. On the fourth day hyosyamim and morphine were given together. Result - died June 5 th, I A.M. The autopsy revealed an ulcerative endocarditis, an old pericarditis with extensive adhesions between heart and pericardium, calcified tubercular deposits in apex of left lung, œdema of both lungs, and a parenchymatous hepatitis; slight meningitis. The vegetations were confined to the mitral valve and were numerous. Some of them had ulcerated. None of the other organs diseased.

Case 2.-John C.; age, I9 years ; native of Ireland; U.S. three years; single; laborer; admitted into the hospital March 6, I889. His temperature at this time was $102^{\circ}$; pulse, 120 ; respiration, 40. His body and limbs were covered with an eruption of a peculiar nature. It was in patches of irregular size and shape; average diameter, 